\title{
Conjecture: Existence of Nash Equilibria in Modern Internet Congestion Control
}

\author{
Ayush Mishra \\ National University of Singapore \\ Singapore \\ ayush@comp.nus.edu.sg \\ Sean $\mathrm{Ng}$ \\ National University of Singapore \\ Singapore \\ seannpn@comp.nus.edu.sg
}

\author{
Jingzhi Zhang \\ Wuhan University \\ China \\ 2017301500155@whu.edu.cn \\ Raj Joshi \\ National University of Singapore \\ Singapore \\ rajjoshi@comp.nus.edu.sg
}

\author{
Melodies Sim \\ National University of Singapore \\ Singapore \\ melodies@comp.nus.edu.sg \\ Ben Leong \\ National University of Singapore \\ Singapore \\ benleong@comp.nus.edu.sg
}

\begin{abstract}
The Internet's congestion control landscape is currently in the midst of an unprecedented paradigm shift. A recent measurement study found that BBR, a congestion control algorithm introduced by Google in 2016, has seen rapid adoption and is deployed at more than $20 \%$ of the Alexa Top 20,000 websites. Encouraging early deployment results from Google, Dropbox and Spotify suggest that BBR could potentially replace traditional loss-based congestion control algorithms like CUBIC. In this paper, we study the interactions between CUBIC and BBR and show that the underlying interactions can be modeled as a normal form game. Our game-theoretic analysis and testbed measurements suggest that while BBR seems to achieve somewhat better performance than CUBIC on the Internet today, this advantage will decrease as the proportion of BBR flows increases. The distribution of congestion control algorithms on the Internet would likely reach a Nash Equilibrium, where no flow has the incentive to switch from CUBIC to BBR, or vice versa. We also found that the distribution of CUBIC and BBR flows in this Nash Equilibrium will be dependent mainly on the size of the bottleneck buffer, and marginally on the RTT distribution of the flows. Our results suggest that the future Internet will likely be more heterogeneous and that buffer sizing will continue to have a significant impact on Internet congestion control.
\end{abstract}

\section{CCS CONCEPTS}

- Networks $\rightarrow$ Transport protocols; Public Internet.

\section{KEYWORDS}

congestion control; measurement study

\section{ACM Reference Format:}

Ayush Mishra, Jingzhi Zhang, Melodies Sim, Sean Ng, Raj Joshi, and Ben Leong. 2021. Conjecture: Existence of Nash Equilibria in

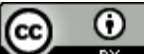

This work is licensed under a Creative Commons Attribution International 4.0 License.

APNet 2021, June 24-25, 2021, Shenzhen, China, China

(c) 2021 Copyright held by the owner/author(s).

ACM ISBN 978-1-4503-8587-9/21/06.

https://doi.org/10.1145/3469393.3469397
}

Modern Internet Congestion Control. In 5th Asia-Pacific Workshop on Networking (APNet 2021) (APNet 2021), June 24-25, 2021, Shenzhen, China, China. ACM, New York, NY, USA, 6 pages. https://doi.org/10.1145/3469393.3469397

\section{INTRODUCTION}

Today, BBR [2] and CUBIC [6] are the two dominant congestion control algorithms on the Internet. The apparent performance benefits and promising deployment results of BBR (and its variant BRRv2) from Google [3], Spotify [5], and Dropbox [9] are likely going to make website operators evaluate if they should adopt the new congestion control algorithm. A recent measurement study found that more than $20 \%$ of the Alexa Top 20,000 websites [8] have already adopted BBR to serve HTTP traffic [15]. Since most of these early adopters are websites that stream video [15], it is plausible that BBR is already contributing to more than $40 \%$ of the downstream traffic on the Internet [20]. This significant change in the Internet's congestion control landscape is notable because BBR represents a paradigm shift in congestion control. While traditional congestion control algorithms like CUBIC relied on packet losses to infer congestion, BBR uses bottleneck bandwidth and RTT estimates. By doing so, BBR avoids filling deep buffers to keep latency low and is more resilient to random packet losses.

There have been many studies on the interactions between CUBIC and BBR. However, unlike previous studies [7, 22, 23] which are mainly focused on flow-level fairness, we attempt to answer the following question: given the aggressive adoption of BBR in recent times, how do we expect the Internet to evolve? Would it be reasonable to expect the whole Internet to switch to $B B R$, and/or its variants, in the near future?

Most recent deployments have cited better throughput as a key reason to switch from CUBIC to BBR $[3,5,9]$. This is not surprising and it seems perfectly plausible that a website would decide to switch from one TCP variant to the other if the switch will result in higher throughput. Hence, we can think of the Internet abstractly as a system of $n$ flows and model it as a normal form game. In game theory, a normal form game is a standard representation of a game where the players have a preference of outcome, which in our case is higher throughput.

Our analysis of such a normal form game suggests that given the characteristics of the interactions between BBR and CUBIC, there must exist a Nash Equilibrium [16] (NE). A Nash Equilibrium in a game is a strategy distribution between the players where no 


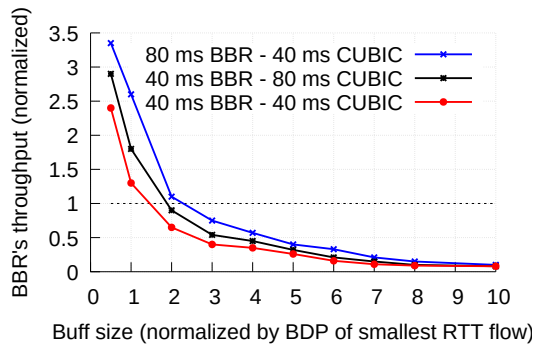

Figure 1: BBR throughput vs. bottleneck buffer size.

player has anything to gain by changing only their strategy. This essentially means that a set of $n$ flows will eventually converge to a distribution of congestion control algorithms, where no flow will have any incentive to switch from CUBIC to BBR, or vice versa because doing so will not lead to an increase in throughput. We verify the existence of such Nash Equilibria and measure the effect of network parameters such as bottleneck link capacity, buffer size, number of flows, and RTT using a set of testbed experiments. Our results suggest that the distribution of congestion control algorithms at the NE depends primarily on the size of the bottleneck buffer and marginally on the RTT distribution of the flows.

\section{KNOWN INTERACTIONS BETWEEN BBR AND CUBIC}

CUBIC and BBR behave very differently when they are in deep buffers. Since CUBIC is loss-based, it will continue to fill a buffer till it experiences a packet loss. On the other hand, BBR caps its $\mathrm{cwnd}$, which prevents it from filling deep buffers. While this behavior is intended to allow BBR to keep the queuing delay low, a competing CUBIC flow will be significantly more aggressive (throughput-wise) and take up a much larger share of the bandwidth. On the other hand, in shallow buffers, CUBIC's throughput will be limited because the cwnd will keep reducing because of the frequent packet drops. Therefore, we make the following general observation which has been verified in previous studies [22,23].

Observation 1. When competing at the bottleneck where the buffer is deep, CUBIC tends to have higher throughput than BBR; the converse is true when the buffer is shallow.

We investigate the impact of RTT with a simple experiment where a CUBIC and a BBR flow share a $20 \mathrm{Mbps}$ bottleneck with varying buffer sizes. Both flows were run simultaneously for two minutes and we plot the steady-state throughput for BBR in Figure 1, normalized against the throughput of the CUBIC flow. As expected, we see that the throughput for BBR is a smooth function of the buffer size. We also see that in each case there exists some buffer size where CUBIC and BBR get an equal share of the bottleneck bandwidth i.e. normalized throughput equals 1. Based on this result, we can make the next observation:

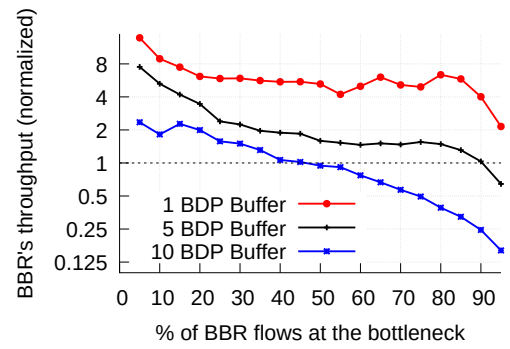

Figure 2: BBR's throughput vs. \% of BBR flows.

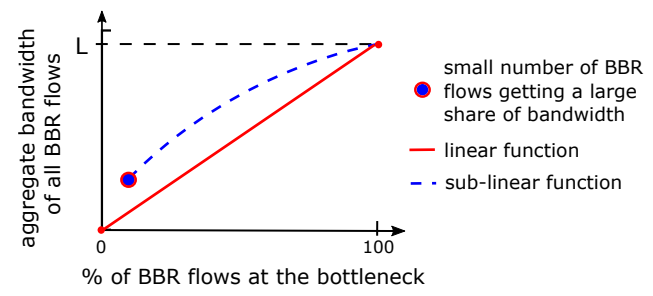

Figure 3: Sub-linear increase in total BBR bandwidth.

Observation 2. When a single BBR flow competes with a single CUBIC flow at a bottleneck, there must exist some threshold bottleneck buffer size $T_{\text {fair }}$ such that when the bottleneck buffer size Buff $<T_{\text {fair }}$, the BBR flow gets higher throughput than the CUBIC flow and when Buff $>T_{\text {fair }}$, the CUBIC flow gets higher throughput than BBR.

Besides buffer size, BBR's throughput also depends on the number of BBR and CUBIC flows at the bottleneck. We simultaneously run 20 CUBIC/BBR flows passing through a $100 \mathrm{Mbps}$ bottleneck. In each iteration, we change the percentage of flows that ran BBR and CUBIC. We plot the per-flow throughput for BBR normalized by the average per-flow throughput of the competing CUBIC flows in Figure 2. All flows had an RTT of $40 \mathrm{~ms}$ and ran for 2 minutes. Our results show that as the percentage of BBR flows in the bottleneck link increase, BBR flows get lower throughput. This behavior is consistent when repeated with different buffer sizes, and the average throughput for the BBR flows can drop below that for the CUBIC flows if the buffer is large and the number of BBR flows is larger than a threshold value. We therefore make the following observation:

Observation 3. As the percentage of BBR flows at the bottleneck increases, the per-flow average throughput of BBR flows at that bottleneck decreases.

Earlier measurement studies had found that a small number of BBR flows can get a disproportionately large amount of bottleneck bandwidth when competing with CUBIC flows [22,23]. As shown in Figure 3, the aggregate bandwidth of BBR flows at a fixed-capacity bottleneck link will increase as the number of flows increases, so the result is a sub-linear increase in total throughput and thus the per-flow throughput would decrease with more BBR flows.

Both BBR and CUBIC congestion control algorithms are also known to be RTT-unfair [10, 22]. CUBIC, being cwnd-based, is hampered in a long RTT path as it can ramp up its cwnd less often 
than another CUBIC flow that has a short RTT path. BBR on the other hand tends to favor flows with longer RTTs as compared to those with shorter RTTs. This is because when BBR becomes cwndlimited, it places twice the BDP (Bandwidth Delay Product) amount of packets in flight. We summarize these findings in Observation 4.

Observation 4. When two BBR flows compete at a bottleneck, the flow with a longer RTT will get higher bandwidth than the flow with a shorter RTT. When two CUBIC flows compete at a bottleneck, the flow with a shorter RTT will get higher bandwidth than the flow with a longer RTT.

\section{MODELLING THE INTERNET AS A NORMAL FORM GAME}

In this section, we will formulate the problem of flows choosing between CUBIC and BBR as a normal form game and analyze it using the observations made in $\S 2$.

To investigate the behavior for a set of $n$ flows, we model each flow as an independent agent that (i) can decide on whether the flow should run BBR or CUBIC; and (ii) knows its current throughput and also the throughput if it were to switch to the alternative algorithm, if all other flows do not change. We assume that websites can determine (ii) by A/B testing between CUBIC and BBR, and that all the flows would prefer to adopt the TCP variant that allows it to achieve higher throughput. While senders generally care about a combination of network parameters, the weights given to each of these parameters may be varied across sites. However, improved throughput was cited as a key factor by all major companies [3, 5, 9] that have reported making a switch from CUBIC to BBR, so for simplicity, we consider only throughput in our analysis. With these assumptions, we effectively have a normal form game, where the websites/senders can maximize throughput via 2 mutually exclusive strategies: (i) BBR; or (ii) CUBIC. In this section, we argue that a Nash Equilibrium should exist.

\subsection{Two-flow game}

Consider a simple network with two senders $S_{1}$ and $S_{2}$ sharing a common bottleneck link. Each sender can pick either CUBIC or BBR. We denote the RTTs of these two flows as $R T T_{1}$ and $R T T_{2}$ respectively. We will assume, without loss of generality, that $R T T_{1}>$ $R T T_{2}$. We will also assume that the bottleneck queue is a simple FIFO queue and does not deploy any other queuing disciplines or AQMs. With two senders and two congestion control algorithms, there can be a total of four possible scenarios or combinations. We enumerate these possible scenarios in Table 1.

Consider the case where the two flows $S_{1}$ and $S_{2}$ pick different algorithms, i.e. scenarios 2 and 3. From Observation 2, we know that for scenario 2, there exists a threshold buffer size $T_{2}$ such that when the bottleneck buffer size Buff $>T_{2}$, the CUBIC flow will get more bandwidth (CUBIC wins), and when Buff $<T_{2}$, the BBR flow will get more bandwidth (BBR wins). Similarly, for scenario 3, there exists a buffer size $T_{3}$ such that when Buff $>T_{3}$, the CUBIC flow wins whereas when Buff $<T_{3}$, the BBR flow wins. In summary, $T_{2}$ and $T_{3}$ are the threshold buffer sizes below which BBR wins against CUBIC in scenarios 2 and 3 respectively.
Table 1: Possible scenarios in a 2-flow system.

\begin{tabular}{ccc}
\hline Scenario & $S_{1}$ & $S_{2}$ \\
\hline 1 & CUBIC & CUBIC \\
2 & CUBIC & BBR \\
3 & BBR & CUBIC \\
4 & BBR & BBR \\
\hline
\end{tabular}

The BBR flow in scenario 3 has a higher RTT than the BBR flow in scenario 2. From Observation 3 we know that a BBR flow with a higher RTT is effectively more aggressive. This means that BBR will be more aggressive in scenario 3 than in scenario 2 and so CUBIC will need a deeper buffer in scenario 3 to tie with BBR. Therefore we conclude that $T_{3}>T_{2}$. This relationship can also be seen in Figure 1.

On this note, we consider 3 different regimes as illustrated in Table 2, where for each scenario we mark the outcome for a sender that gets the higher bandwidth as 'W' (winner) and the outcome for the other sender as 'L' (loser). A sender choosing either CUBIC or $\mathrm{BBR}$ as their congestion control algorithm is denoted with the letter $\mathrm{C}$ and $\mathrm{B}$, respectively, and the winning congestion control variant in each case has also been highlighted for easy analysis. In particular, we see that:

(1) When Buff $<T_{2}$ : $S_{1}$ will definitely pick BBR regardless of what $S_{2}$ decides to pick. In economic terms, $B$ is a dominant strategy for $S_{1}$. $S_{2}$ will pick the algorithm that achieves the higher throughput and we get the Nash Equilibrium $(B, *)$.

(2) When $T_{2}<B u f f<T_{3}$ : B is still a dominant strategy for $S_{1}$ and we again have the Nash Equilibrium $(B, *)$.

(3) When $T_{3}<B u f f$ : $C$ is now a dominant strategy for $S_{2}$ and we end up with a Nash Equilibrium $(*, C)$.

By enumerating all possible scenarios, we have effectively shown that unique Nash Equilibria must exist for a 2-flow system. We verified our predictions with experiments where two flows compete at a $20 \mathrm{Mbps}$ bottleneck with $R T T_{1}=80 \mathrm{~ms}$ and $R T T_{2}=40 \mathrm{~ms}$. For buffer sizes less than twice the BDP of the smaller flow, the NE was at $(\mathrm{B}, \mathrm{B})$ and for all other buffer sizes up to $10 \mathrm{BDP}$ the NE was at (B,C).

\subsection{Conjecture for $\boldsymbol{n}$ flows}

While we can model a system with $n$ flows using the methodology in §3.1, enumerating all possible scenarios is not a feasible approach to prove the existence of Nash Equilibria. However, some forms of normal form games have been studied extensively and proven to have a pure strategy Nash Equilibrium $[14,19]$. These games show unique properties like continuity and boundedness of pay-offs, and measurability of outcome, that can be easily extended to our setting. It remains as future work to check if it is possible to model an $n$-flow system as one of these normal form games so that we can apply known results.

The complexity for a $n$-flow system arises from the exponentiation of the number of scenarios and also the fact that flows can have different RTTs, which makes it intractable to enumerate all possibilities. However, there are two key observations from our experiments that inspire us to propose Conjecture 1 below: (1) As the proportion of BBR flows increases, the gain in throughput will decrease (cf. Observation 3 in $\S 2$ ). When the proportion of BBR 
Table 2: Outcomes in a two-flow game. $\left(R T T_{1}>R T T_{2}\right.$, winning strategies are highlighted $)$

\begin{tabular}{|c|c|c|c|c|c|c|c|c|c|c|c|c|}
\hline & \multicolumn{4}{|c|}{ Buff $<T_{2}$} & \multicolumn{4}{|c|}{$T_{2}<$ Buff $<T_{3}$} & \multicolumn{4}{|c|}{$T_{3}<$ Buff } \\
\hline \multirow[t]{2}{*}{ Scenario } & \multicolumn{2}{|c|}{ Strategies } & \multicolumn{2}{|c|}{ Outcome } & \multicolumn{2}{|c|}{ Strategies } & \multicolumn{2}{|c|}{ Outcome } & \multicolumn{2}{|c|}{ Strategies } & \multicolumn{2}{|c|}{ Outcome } \\
\hline & $S_{1}$ & $S_{2}$ & $S_{1}$ & $S_{2}$ & $S_{1}$ & $S_{2}$ & $S_{1}$ & $S_{2}$ & $S_{1}$ & $S_{2}$ & $S_{1}$ & $S_{2}$ \\
\hline 1 & C & C & $\mathrm{L}$ & W & C & c & $\mathrm{L}$ & W & $\mathrm{C}$ & C & $\mathrm{L}$ & W \\
\hline 2 & $\mathrm{C}$ & BB & $\mathrm{L}$ & W & C & B & W & $\mathrm{L}$ & C & B & W & $\mathrm{L}$ \\
\hline 3 & B] & C & W & $\mathrm{L}$ & B & C & W & $\mathrm{L}$ & B & C & $\mathrm{L}$ & W \\
\hline 4 & B & B & $\mathrm{W}$ & $\mathrm{L}$ & B & B & $\mathrm{W}$ & $\mathrm{L}$ & (B) & B & W & $\mathrm{L}$ \\
\hline Nash Equilibrium & & $(B$ & & & & $(B$ & & & & & & \\
\hline
\end{tabular}

flows increases beyond a threshold, the average throughput of the BBR flows tends to drop below that of the remaining CUBIC flows (see Figure 1). (2) When the buffer is shallow, BBR is a dominant strategy for flows with large RTTs; similarly, when the buffer is deep, CUBIC is a dominant strategy for flows with short RTTs.

Conjecture 1. In a system with $n$ flows that can choose to run either CUBIC or BBR, there will be at least one Nash Equilibrium.

\section{EVALUATION}

In this section, we present the results of our testbed experiments that: (i) suggest that NE exists for $n$ flows (for $n>2$ ), and (ii) provide some insights into how RTT and buffer size affect the NE. These experiments serve as validation of Conjecture 1 in a limited state space and do not attempt to replicate all the network interactions possible on the Internet (since that would be impossible).

Testbed setup. Our testbed setup consists of two servers connected by a $1 \mathrm{Gbps}$ link. We use Mahimahi [17] to spawn individual iperf senders with different RTTs. We use nfqueue [18] to create a bottleneck with a specified link capacity and buffer size.

Methodology. For each network setting, we run 3 trials of all the $2^{n}$ possible combinations of the $n$ senders running either CUBIC or BBR. In each trial, the senders send data for 2 minutes and we record their average per-flow throughput. To identify the NE, we enumerate all the combinations and check if there is any combination such that no individual flow in that combination can achieve higher throughput if it switches to the other TCP variant (all other flows remaining fixed). Since the number of combinations increases exponentially with $n$, we have only been able to do this exercise for up to a maximum of 12 flows. It is clear that we will not be able to prove Conjecture 1 with experiments. However, in this section, our goal is to verify that the Conjecture holds for some $n>2$ in practice and that there are no obvious counter-examples. In all our experiments, we found a NE as our Conjecture suggests.

Nash Equilibria Found. In all cases we found exactly one NE. All these NE share a common property that allows us to specify it succinctly with a single number: the proportion of CUBIC flows. In particular, we observe that in any NE combination of $n$ flows, if we sort the flows by their RTTs, then it is always possible to write the NE combination where the first $m$ flows pick CUBIC, and the remaining $(n-m)$ flows pick BBR. For example, in a 6-flow experiment, where flows have an RTT distribution of $(20 \mathrm{~ms}, 20 \mathrm{~ms}$, $50 \mathrm{~ms}, 50 \mathrm{~ms}, 80 \mathrm{~ms}, 80 \mathrm{~ms}$ ), if the NE exists when $50 \%$ of the flows pick CUBIC the NE combination will look like (C, C, C, B, B, B).
That is, all the $20 \mathrm{~ms}$ flows and one $50 \mathrm{~ms}$ flow picking CUBIC and all the $80 \mathrm{~ms}$ flows and one $50 \mathrm{~ms}$ flow picking BBR. This indicates that smaller RTT flows prefer CUBIC over BBR at the NE. We use this property to represent a NE combination with a single number - the percentage of flows running CUBIC in the NE combination $(m / n \times 100)$. The CUBIC flows are always the flows with the smallest RTTs in the combination. This is not surprising, since smaller RTT flows perform worse than larger RTT flows when running BBR and vice versa for CUBIC.

\subsection{Effect of buffer size}

In Figure 4, we present the NE when 6, 9, and 12 flows compete at 3 different bottleneck links with speeds of 20, 50 and $100 \mathrm{Mbps}$. In each experiment, the flows are divided into 3 groups with RTTs of $20 \mathrm{~ms}, 50 \mathrm{~ms}$ and $80 \mathrm{~ms}$, respectively. We let these flows compete in the common bottleneck with different buffer sizes and record the congestion control algorithm distribution at the NE in each case. In general, when the buffers are deeper, more flows would pick CUBIC over BBR. At very large buffer sizes, the NE generally occurs near a fifty-fifty split between CUBIC and BBR.

\subsection{Effect of other network parameters}

Our experimental results in Figure 4 also suggest that link capacity and the number of flows have little to no effect on the distribution of CUBIC and BBR in the NE. It is plausible that because we size the bottleneck buffer based on the BDP of the smallest RTT flow (smallest RTT multiplied by the bottleneck link capacity), any differences caused by an increase in link capacity are normalized by the proportional increase in the buffer size. While our experiments suggest that the number of flows does not have much effect on the distribution of congestion control algorithms in the NE combination, this could also be an artifact of our limited number of experiments.

In Figure 5, we plot the results for two different RTT distributions. We found that when the flows have larger RTTs, the NE has a marginally lower percentage of flows picking CUBIC. In other words, the RTT distribution does have a marginal impact on the NE.

\section{DISCUSSION AND FUTURE WORK}

Our experimental results suggest that with realistically sized (at least $1 \mathrm{BDP}$ ) buffers, the Nash Equilibrium for $n$ flows will be a mix of both CUBIC and BBR flows, and not one where all the flows are BBR. While recent studies $[7,13]$ have shown BBR to have better link utilization and lower and less variable delay, and BBR's recent 


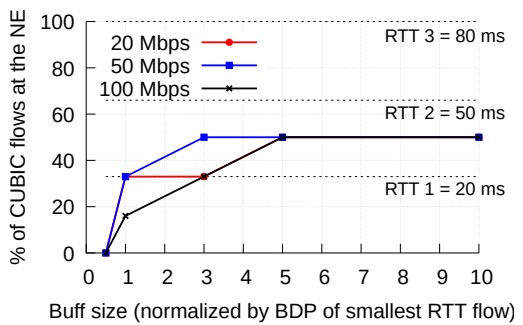

(a) 6 flows

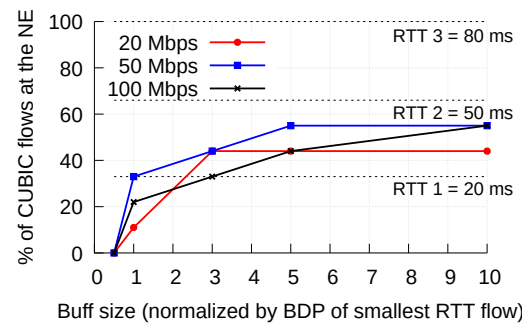

(b) 9 flows

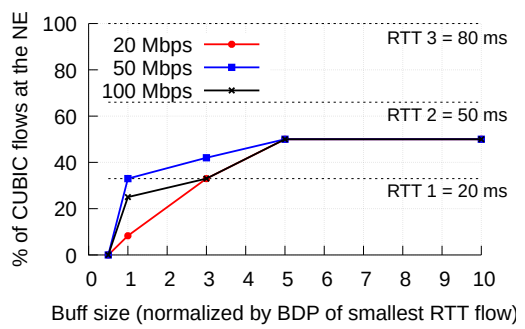

(c) 12 flows

Figure 4: The effect of link capacity and number of flows on the Nash Equilibrium. RTTs $20 \mathrm{~ms}, 50 \mathrm{~ms}$ and $80 \mathrm{~ms}$.

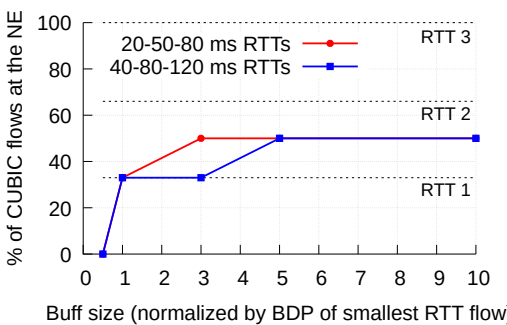

(a) 6 flows, 50 Mbps

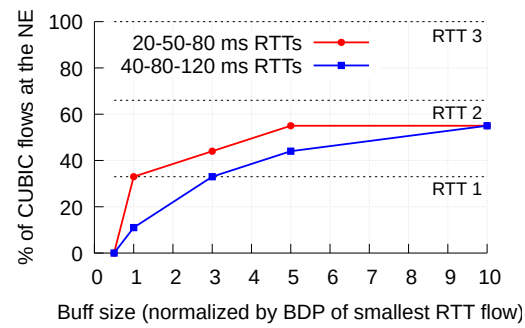

(b) 9 flows, 50 Mbps

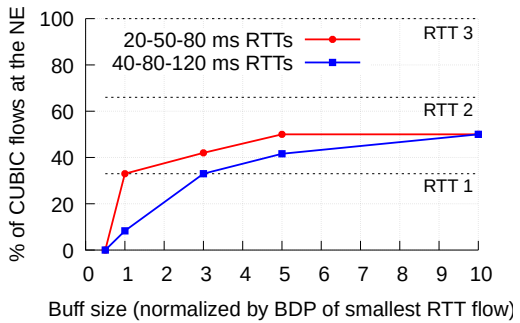

(c) 12 flows, $50 \mathrm{Mbps}$

Figure 5: The effect of the RTT distribution on the Nash Equilibrium.

deployments $[3,5,9]$ have shown good performance, the corollary is that we would expect the benefits of deploying BBR to be reduced as more flows switch from CUBIC to BBR.

The future of Internet congestion control. Notwithstanding the rapid adoption of BBR in recent years [15], our results suggest that CUBIC is not about to disappear or to be completely replaced anytime soon. Instead, the Internet is likely to become more heterogeneous. This means that existing networks and middleware will need to work well with both traditional loss-based TCP, as well as with BBR and its variants.

The best is yet to come. We note that there is already work being done on BBRv2 [4], which is a less aggressive version of BBR. HTTP/3.0 is going to adopt QUIC $[1,11]$ as its transport stack. With QUIC, the entry barrier to developing and deploying newer congestion control algorithms is even lower. It remains to be seen how the nature of the NE will change in such a heterogeneous environment. Even if our Conjecture in $\S 3.2$ is true for $n$ flows where they can be either CUBIC or BBR, it is not clear if it would hold for any $n$ flows if there are no restrictions on the choice of the congestion control algorithm. It remains as future work to investigate if and how a NE will exist in the presence of BBRv2 and any future QUIC-based congestion control algorithms.

The Internet may not follow economic game theory. We have applied concepts from game theory to model the evolution of Internet congestion control. It has also provided us with a principled way to analyze and reason about the interactions between CUBIC and BBR. However, it is also clear that the Internet does not necessarily follow economic game theory perfectly. For example, it is quite clear that CUBIC is a dominant strategy when competing with New Reno, i.e. one always achieves higher throughput since CUBIC is much more aggressive than New Reno. Despite this, there are still a small number of websites on the Internet today that continue to run New Reno [15]. This phenomenon could potentially be attributed to the indifference of some websites to network performance or the influence of some other extraneous factors. Such phenomenon contradicts what game theory would predict.

Uniqueness of the Nash Equilibria. In all the experiments, we found exactly one NE. We were tempted to conjecture in $\S 3.2$ that not only does a NE exist, but that it would be unique. We resisted doing so because of the large space of possibilities when there are a large number of flows and many different RTTs. A Nash Equilibrium is like a local minimum. It remains as an open question whether an $n$-flow system could have multiple Nash Equilibria, and if so, what determines whether there are multiple $\mathrm{NE}$ or a unique one.

Impact of workloads, AQMs, and multi-hop paths Admittedly, the analysis done in this paper does not represent the conditions at an Internet scale. It remains to be seen how the normal form game would be affected by flow sizes and lengths, and how the interaction between CUBIC and BBR will change when the bottlenecks move or deploy AQMs like RED and CoDel.

\section{RELATED WORK}

To the best of our knowledge, there have not been any previous game-theoretic studies on the interactions between CUBIC and BBR. However, there have been several measurement studies that try to measure and model the interactions of BBR with itself and with traditional loss-based TCP like CUBIC. These works focus on flow-level fairness and general network metrics like throughput, utilization, and delay.

Scholz et al. [21] and Miyazawa et al. [24] showed that sharing a bottleneck with loss-based protocols such as CUBIC causes BBR to measure an over-inflated RTT as loss-based protocols tend to 
fill up buffers. This drastically increases BBR's congestion window. The net result is that the throughput for BBR and CUBIC will fluctuate cyclically. While one can näively expect CUBIC to become a dominant strategy for very large buffers, the RTT over-estimation associated with BBR can explain why some BBR flows are still able to remain competitive and the NE does not end up being all flows running CUBIC.

Similar phenomena were also reported by Hock et al. [7] who showed that BBR's over-estimation of RTT is aggravated when multiple BBR flows share a bottleneck and over-estimate their fair share of the bandwidth. They attribute this to the implementation of BBR where each flow will measure the maximum available bandwidth over 10 RTTs. This results in the sum of derived throughput to be always greater than the actual maximum bandwidth of the bottleneck, causing persistent queues to build up at the bottleneck.

More recently, Ware et al. proposed a model to analyze how BBR can be detrimental towards CUBIC when the two of them share a bottleneck [23]. While their results assume that the network has very deep bottleneck buffers, their finding that BBR gets cwnd-limited when competing with other flows explains the RTTunfairness observed between BBR flows.

\section{CONCLUSION}

In this paper, we investigate the potential evolution of Internet congestion control by modeling it as a normal form game. Through analysis and testbed experiments, we show that if all flows were either CUBIC or BBR, Nash Equilibria (NE) exists for a small number of flows and we conjecture that a Nash Equilibrium will exist in general for larger systems like the Internet. Our results suggest that under realistic network settings, this Nash Equilibrium is likely a heterogeneous mix of CUBIC and BBR flows. Furthermore, the relative proportions of CUBIC and BBR flows that make up the NE will depend primarily on the size of the bottleneck buffer and marginally on the RTT distribution of the flows, with smaller RTT flows generally picking CUBIC.

The existence and the nature of these Nash Equilibria will impact how we can expect the Internet's congestion control landscape to evolve over the next few years and how it affects the performance benefits associated with the adoption of new congestion control algorithms like BBR. In particular, our findings effectively debunk the commonly held notion that switching to BBR would improve throughput [12]. We also suspect that it is unlikely that the entire Internet will switch from CUBIC to BBR and/or its variants in the near future.

\section{ACKNOWLEDGMENTS}

This work was supported by the Singapore Ministry of Education grant T1 251RES1917.

\section{REFERENCES}

[1] Ed. M. Bishop. 2021. Hypertext Transfer Protocol Version 3 (HTTP/3). (2021). https://tools.ietf.org/html/draft-ietf-quic-http-34

[2] Neal Cardwell, Yuchung Cheng, C Stephen Gunn, Soheil Hassas Yeganeh, and Van Jacobson. 2017. BBR: congestion-based congestion control. Commun. ACM 60, 2 (2017), 58-66.

[3] Neal Cardwell, Yuchung Cheng, C. Stephen Gunn, Soheil Hassas Yeganeh, Van Jacobson, and Amin Vahdat. 2017. TCP BBR congestion control comes to GCP your Internet just got faster. (2017). https://tinyurl.com/yc7bd9jk
[4] Neal Cardwell, Yuchung Cheng, Soheil Hassas Yeganeh, Ian Swett, Victor Vasiliev, Priyaranjan Jha, Yousuk Seung, Matt Mathis, and Van Jacobson. 2019. BBR v2 A Model-based Congestion Control. (March 2019). https://datatracker.ietf.org/ meeting/104/materials/slides-104-iccrg-an-update-on-bbr-00

[5] Erik Carlsson and Eirini Kakogianni. 2018. Smoother Streaming with BBR. (2018). https://tinyurl.com/yyt5tbhd

[6] Sangtae Ha, Injong Rhee, and Lisong Xu. 2008. CUBIC: A New TCP-friendly High-speed TCP Variant. SIGOPS Operating Systems Review 42, 5 (2008), 64-74.

[7] Mario Hock, Roland Bless, and Martina Zitterbart. 2017. Experimental Evaluation of BBR Congestion Control. In Proceedings of ICNP.

[8] Alexa Internet Inc. 2018. The Top 500 websites on the Internet. (2018). https: //www.alexa.com/topsites

[9] Alexey Ivanov. 2019. Evaluating BBRv2 on the Dropbox Edge Network. (2019). https://tinyurl.com/yyrs68pp

[10] Tomoki Kozu, Yuria Akiyama, and Saneyasu Yamaguchi. 2013. Improving RTT Fairness on CUBIC TCP. In Proceedings of the International Symposium on Computing and Networking.

[11] Adam Langley, Alistair Riddoch, Alyssa Wilk, Antonio Vicente, Charles Krasic, Dan Zhang, Fan Yang, Fedor Kouranov, Ian Swett, Janardhan Iyengar, Jeff Bailey, Jeremy Dorfman, Jim Roskind, Joanna Kulik, Patrik Westin, Raman Tenneti, Robbie Shade, Ryan Hamilton, Victor Vasiliev, Wan-Teh Chang, and Zhongyi Shi. 2017. The QUIC Transport Protocol: Design and Internet-Scale Deployment. In Proceedings of SIGCOMM.

[12] Frederic Lardinois. 2017. Google Cloud gets a new networking algorithm that boosts internet throughput. (2017). https://tinyurl.com/yjswop2z

[13] Feng Li, Jae Won Chung, Xiaoxiao Jiang, and Mark Claypool. 2018. TCP CUBIC versus BBR on the Highway. In Proceedings of PAM.

[14] Andreu Mas-Colell. 1984. On a theorem of Schmeidler. Fournal of Mathematical Economics 13, 3 (1984), 201-206.

[15] Ayush Mishra, Xiangpeng Sun, Atishya Jain, Sameer Pande, Raj Joshi, and Ben Leong. 2019. The Great Internet TCP Congestion Control Census. In Proceedings of SIGMETRICS.

[16] John F. Nash. 1950. Equilibrium points in n-person games. Proceedings of the National Academy of Sciences (1950).

[17] Ravi Netravali, Anirudh Sivaraman, Somak Das, Ameesh Goyal, Keith Winstein, James Mickens, and Hari Balakrishnan. 2015. Mahimahi: Accurate Record-andReplay for HTTP. In Proceedings of ATC.

[18] Netfilter Organization. 2019. libnetfilter_queue. (2019). https://bit.ly/2HimY17

[19] Kali P. Rath. 1992. A Direct Proof of the Existence of Pure Strategy Equilibria in Games with a Continuum of Players. Economic Theory 2, 3 (1992), 427-433.

[20] Canada Sandvine Inc. Waterloo, ON. 2018. The 2018 Global Internet Phenomena Report. (2018). https://www.sandvine.com/phenomena

[21] Dominik Scholz, Benedikt Jaeger, Lukas Schwaighofer, Daniel Raumer, Fabien Geyer, and Georg Carle. 2018. Towards a Deeper Understanding of TCP BBR Congestion Control. In Proceedings of IFIP.

[22] Y. Tao, J. Jiang, S. Ma, L. Wang, W. Wang, and B. Li. 2018. Unraveling the RTTfairness Problem for BBR: A Queueing Model. In Proceedings of GLOBECOM.

[23] Ranysha Ware, Matthew K. Mukerjee, Srinivasan Seshan, and Justine Sherry. 2019. Modeling BBR's Interactions with Loss-Based Congestion Control. In Proceedings of IMC.

[24] Kouto Miyazawa; Kanon Sasaki; N. Oda; S. Yamaguchi. 2018. Cycle and Divergence of Performance on TCP BBR. In Proceeding of CloudNet. 